\title{
Sciendo ANALYSIS OF MARINE PISTON ENGINE INJECTORS DYNAMIC STATE FOR THE NON-INVASIVE DIAGNOSTICS PURPOSE
}

DOI 10.2478/ntpe-2018-0060

\author{
MSc. Marek Dudziński \\ MSc. Marcin Kluczyk \\ Polish Naval Academy, Poland
}

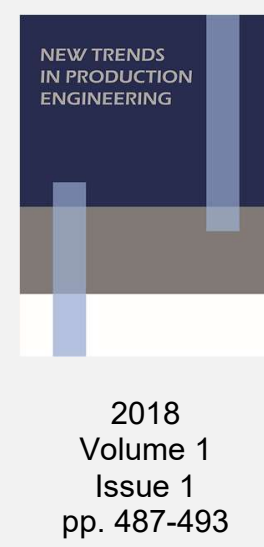

\begin{abstract}
The paper presents results of measurements conducted during multi-stage studies on injectors of WOLA $72 \mathrm{H} 6$ engine. The research was conducted to determine the technical condition of the injectors during their operation. As a result of the injector modeling in the CAD environment, the resonant frequencies of the examined element were obtained. The next stage of the study was a stand-alone tests with the simultaneous recording of vibration parameters. The aim of records was to link the vibration parameters with the technical condition of the tested injectors. The study series was finished with measurements on a real object test stand.
\end{abstract}

Keywords: injectors, vibrations, marine piston engines, technical state

\section{INTRODUCTION}

Operation of marine piston engines on merchant ships and naval vessels involves the need for diagnostic tests. In principle, two strategies for the diagnosis of marine piston engines are discussed. The first one is on-line measurements that give the greatest opportunity to assess actual technical condition, its causes, and future forecasts. This is primarily that they give an ability to identify trends of measured parameters. However, most of technical diagnostics is carried out occasionally. These tests are performed on an ad-hoc basis depending on the symptoms indicating abnormal operation. It is very important to shorten the diagnostic time of engines. The need to stop them will result in shutdown from the operation of the vessel. This cause to the ship owner to bear significant financial losses, and the warships are unable to carry out their combat duties. These aspects have led to research into the possibility of determining the dynamic state of injectors for marine piston engines in the scope of noninvasive diagnostics, which can be carried out without dismantling the engine components. Moreover, in most cases during its normal operation. Similar issues have already been explored by national centers (Czechyra, 2008, Grządziela and Kluczyk, 2017, Kluczyk and Grządziela, 2017, Monieta, 2013, Ranachowski and Bejger, 2005), as well as foreign (Antoni et al., 2002, Carlucci et al., 2006, Elamin et al., 2010, Jianmina et al., 2011, Pflumm et al., 2012, Moshou et al., 2014, Wang, 2009), but there is still a significant gap. This can be completed by the method proposed by the authors.

\section{THEORETICAL ANALYSIS OF THE PROPOSED METHOD USEFULNESS}

The possibility of evaluating the technical condition of the injectors on the basis of their vibration parameters was started from theoretical considerations. In most cases, the mid- and high-speed marine piston engines injectors are mounted under the timing covers, so the choice of vibration transducers mounting points is on the elements of cylinder heads (or monoblocks). Due to the often significant distance of the measuring point from the source of vibration and the material damping occurrence, an attempt to determine the effect of injection pressure on the impact energy of the needle during the injection process was made. The length of the injector spring is assumed when it is closed as (Jianmina et al., 2011).

$$
y_{c}=\frac{F_{c}}{k}
$$


where:

$F_{c^{-}}$- force caused by pressure effect on the surface of the needle at injection start time (injector is still closed);

$y_{c}$-spring length in closed position;

$k$-spring stiffness;

$$
y_{o}=\frac{F_{o}}{k}
$$

where:

$F_{o}$ - force caused by pressure effect on the surface of the needle at fully open position;

$y_{o}$ - spring length in fully open position;

$$
E_{p s}=E_{k}+E_{p s}{ }^{\prime}
$$

where:

$E_{p s}$ - spring potential energy in fully open position;

$E_{p s}{ }^{\prime}$ - spring potential energy in closed position;

$E_{k}$ - kinetic energy of system;

$$
\begin{gathered}
\frac{k \cdot y_{c}{ }^{2}}{2}=\frac{m \cdot v^{2}}{2}+\frac{k \cdot y_{o}{ }^{2}}{2} \\
E_{k}=\frac{m \cdot v^{2}}{2}=\frac{F_{o}{ }^{2}}{2 k}-\frac{F_{c}{ }^{2}}{2 k}=\frac{F_{o}{ }^{2}-F_{c}{ }^{2}}{2 k}
\end{gathered}
$$

During theoretical analysis, the losses resulting from friction were omitted. Analysis of the equations proofs that variations in injection pressure must result in changes of needle kinetic energy striking the seat, and therefore the vibration characteristics of the injector also must be changed.

\section{MODELING}

In order to determine the resonance frequency of the investigated element, which is supposed to achieve the highest values during measurements, an attempt was made to perform a modal analysis of the injector. Based on the technical documentation of the injector and on the basis of its measurements, a 3D model has been made in the CAD program. High dimensional accuracy was ensured and the material properties of all components were given. Selected modeled injector parts were presented in Figures 1 and 2.

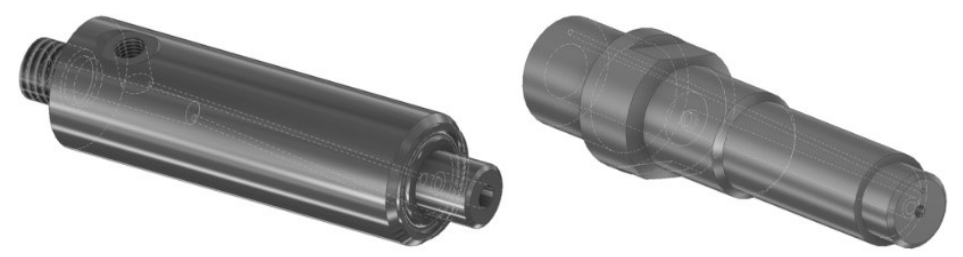

Fig. 1. Upper and lower injector body.

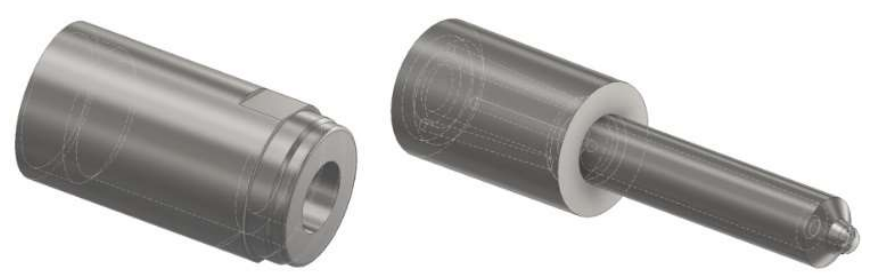

Fig. 2. Clamping nut and nozzle.

Modeling of individual parts was done with feed and overflow fuel channels (Fig. 3). All technological orifices had been also modeled. The next step was assembling injector individual parts, taking into account the internal structure, which was highlighted in colors for better distinguishability and better visibility of the parts. After modeling all components, they were given the appropriate constraints and limitations - resulting from the construction of the injector. The composite model is shown below, while the quarter-section illustrates the inner structure of the injector. 


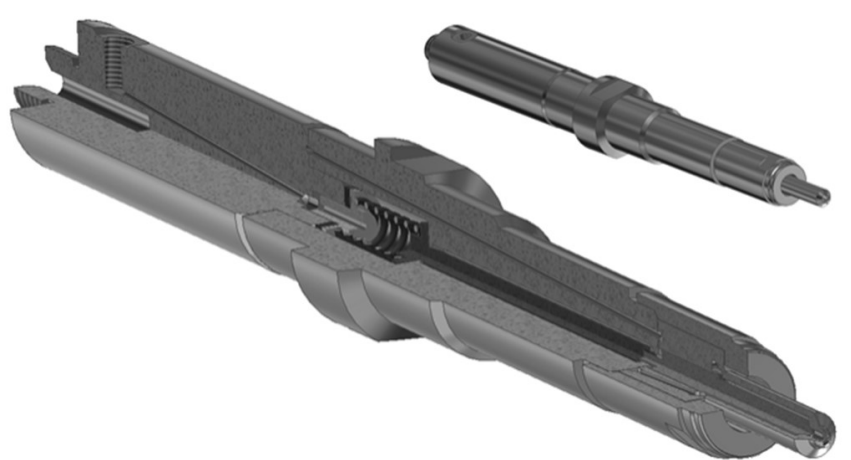

Fig. 3. Injector quarter-section and composite model view.

The model was used to run a modal simulation to determine the resonant frequency of the injector. In order to maintain the correct simulation, it was important to fix the injector in place of the natural mounting on the engine. Results of the modal analysis for the resonant frequency range $0-7 \mathrm{kHz}$ was shown in Figure 4.
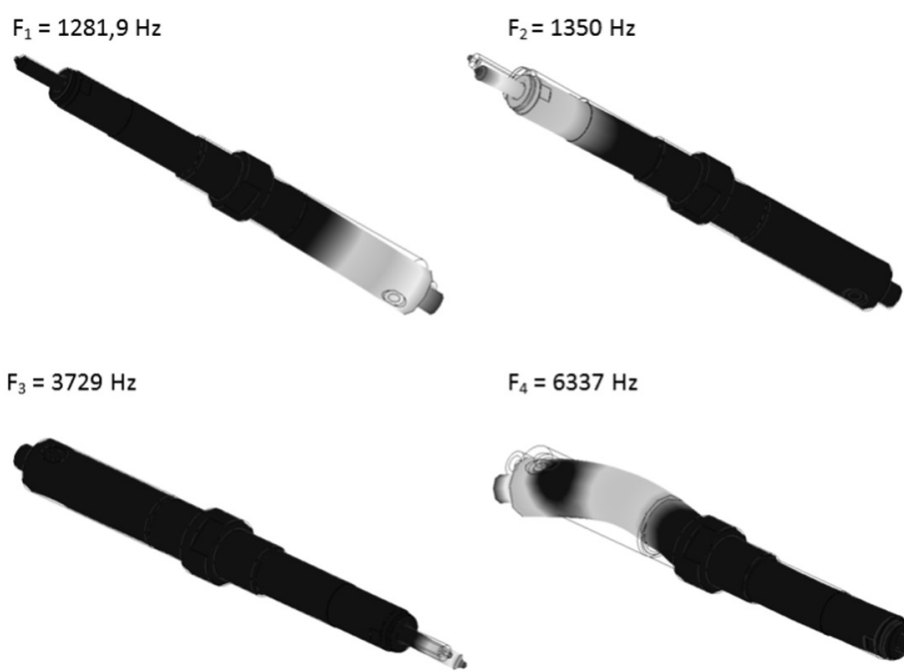

$F_{4}=6337 \mathrm{~Hz}$

Fig. 4. Results of injector modal analysis for frequency range 0-7 kHz.

\section{VERIFICATION TESTS}

Verification tests were started by measuring vibration parameters of Wola $52 \mathrm{H} 6$ engine injectors at the injector control station - Fig. 5.

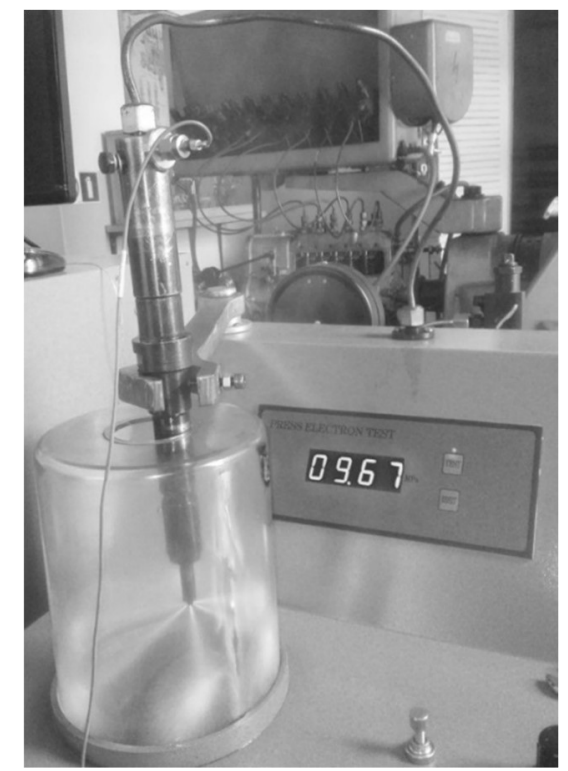

Fig. 5. Wola $52 \mathrm{H} 6$ engine injector at the injectors control station. 
The measurements were conducted in four stages for different fuel injection pressures. Nominal pressure for this type of injectors is $19.4 \mathrm{MPa}$. Three other reduced values of pressure were also adjusted to simulate different degrees of degradation of the technical condition of the (18.8 MPa, 16.7 MPa and 15.7 MPa).

Firstly analysis of vibrations accelerations amplitude-frequency spectra were conducted Figure 6 . The analyzes were conducted in the band up to $6.4 \mathrm{kHz}$, while comparing the values of the average arms calculated in the range of 1,2 to $2,2 \mathrm{kHz}$. The analyzes used a fast Fourier transform with overriding maximum values.

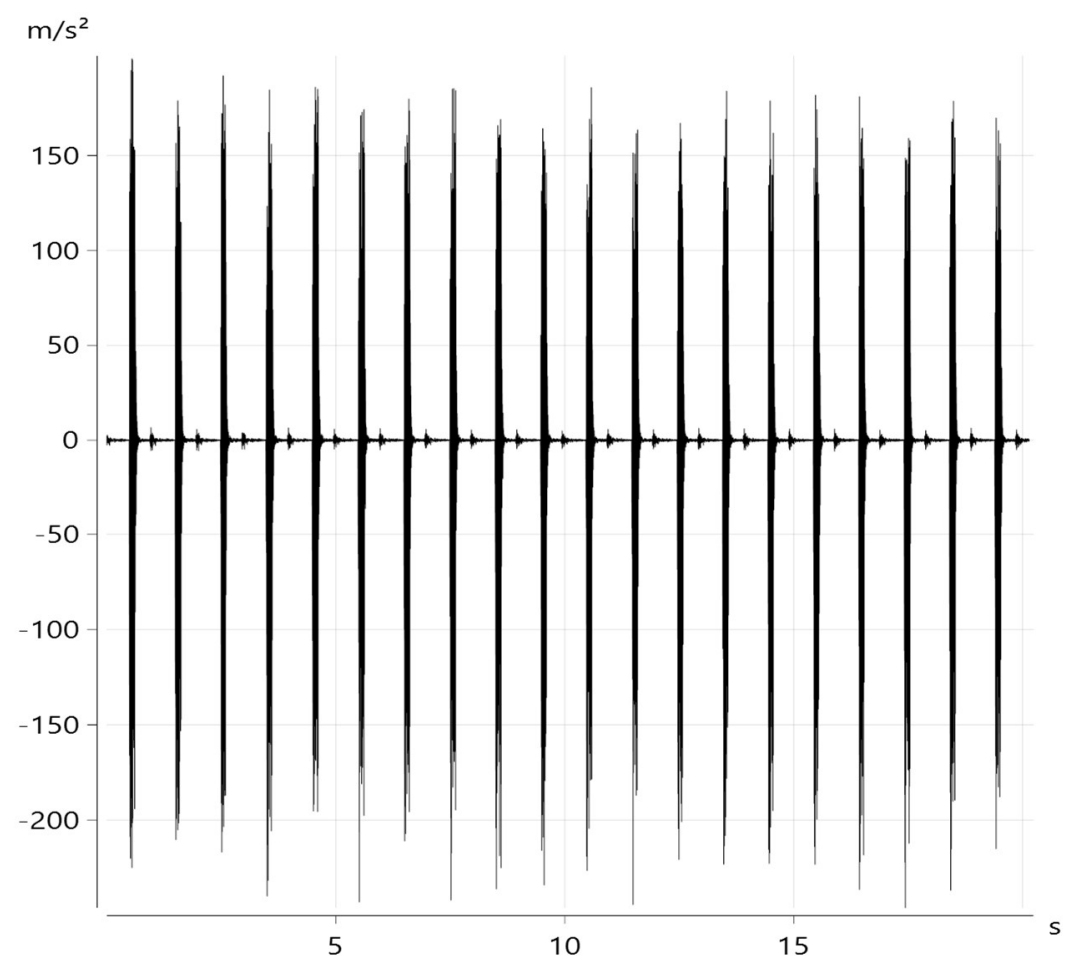

Fig. 6. Wola $52 \mathrm{H} 6$ engine injector accelerations in time domain at the injector test station for a nominal injection pressure of 19.4 MPa.

Analyzing the spectra presented in Figure 7, there is a visible relationship between the opening pressure of the injector and the mean arms value in the expected resonance frequency range. The observed spectrum was also subjected to single integration to obtain the amplitude-frequency spectra of the vibration velocity shown in Figure 8.

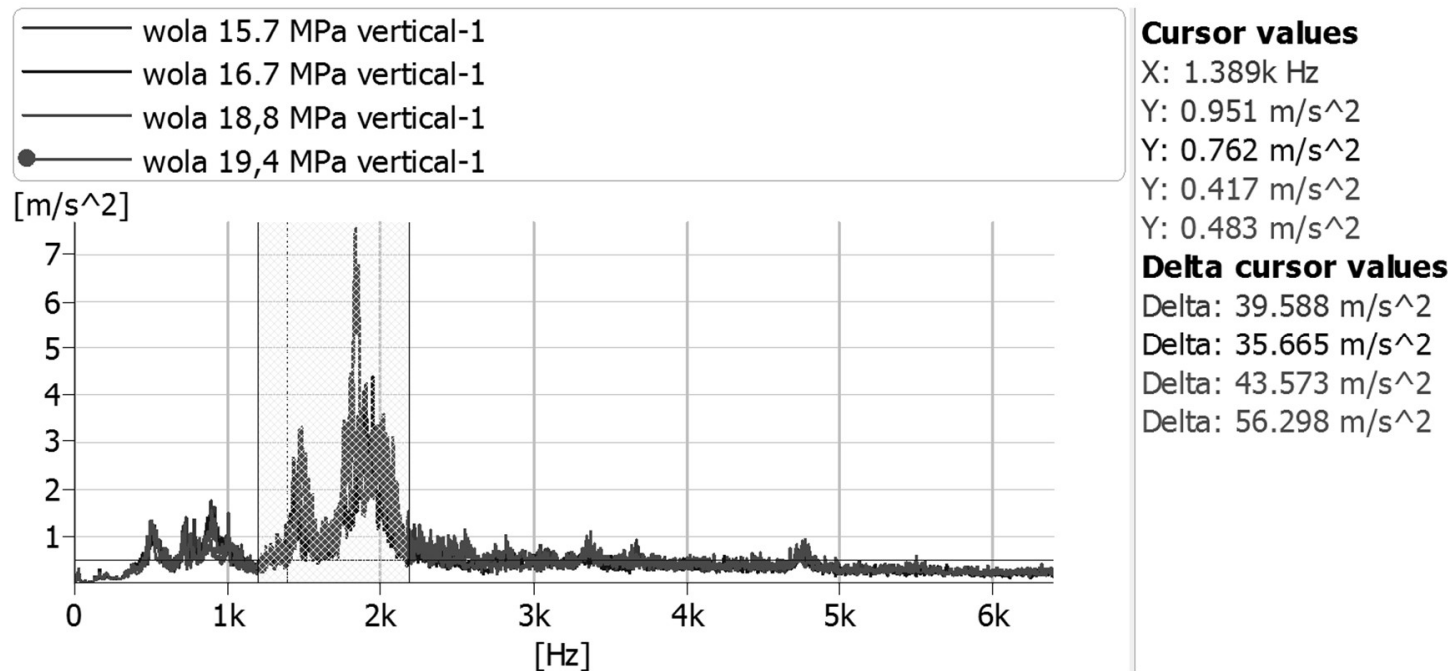

Fig. 7. The vibrations accelerations amplitude-frequency spectra of the injector measured vertically for different opening pressures. 


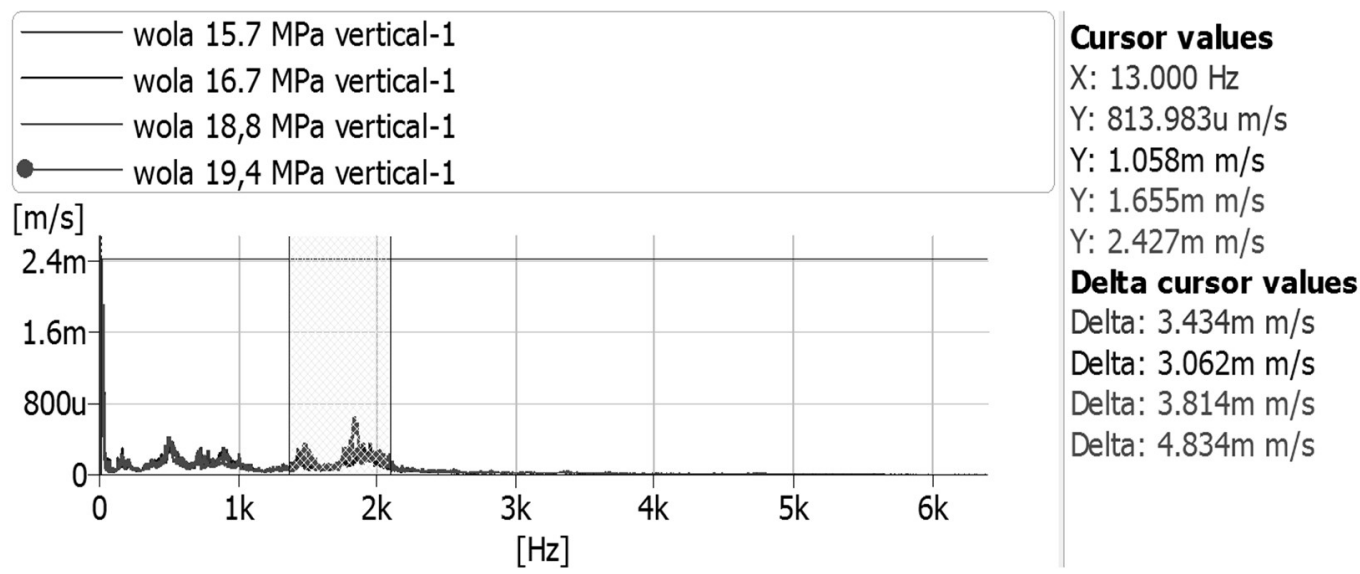

Fig. 8. The vibrations velocities amplitude-frequency spectra of the injector measured vertically for different opening pressures.

In this case, there are the same dependencies as for vibrations accelerations spectra. Values of the vibration parameters for the nominal injection pressure obtain the highest values. They decrease with the decrease in injection pressure, up to the lowest pressure. At this pressure the values of the vibration parameters increase slightly, this should be explained by a few times the needle stroke in one injection.

\section{MEASUREMENTS AT ENGINE TEST STAND WOLA 52 H6}

The next step was measurements at the Wola $52 \mathrm{H} 6$ engine laboratory stand. Measurements were made for an engine load of $140 \mathrm{Nm}$ and two different rotational speeds of $1100 \mathrm{rpm}$ and $1500 \mathrm{rpm}$ respectively. This is an engine that does not have indicator valves, so it is not possible to measure the pressure inside the cylinder. The vibration acceleration spectra are shown in Figures 9 and 10 and the vibration velocity spectra in Figures 11 and 12 .

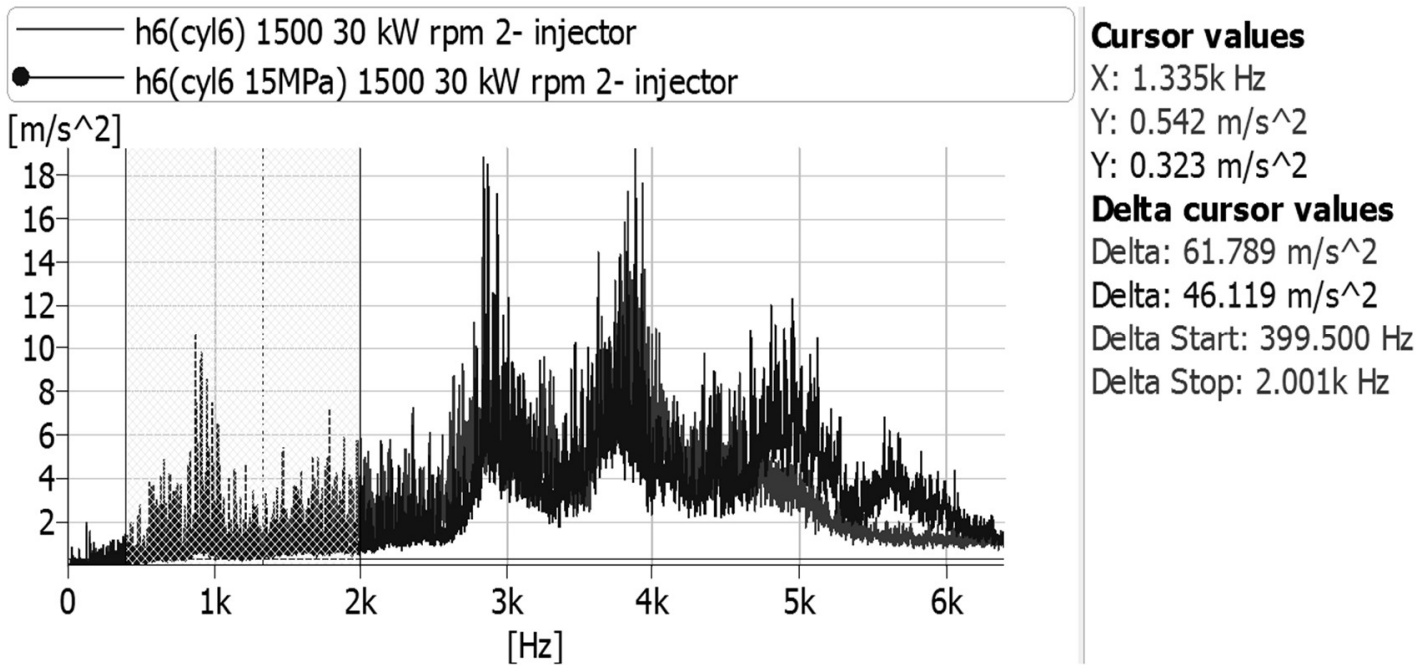

Fig. 9. The vibrations accelerations amplitude-frequency spectra of the injector measured vertically during operating on engine at rotational speed of $1500 \mathrm{rev} / \mathrm{min}$ for two different injection pressures.

It could be stated that there are clearly differences in the distribution of the frequency components of the acceleration and velocity spectra of the injector vibration. This of course results from the interference of the vibration signals generated by the injector and by the running engine and additionally by all its suspension mechanisms. However, despite such significant changes, it is still quite easy to notice in the spectra the differences between the injector operation with the nominal pressure and the reduced pressure of the fuel injection. 


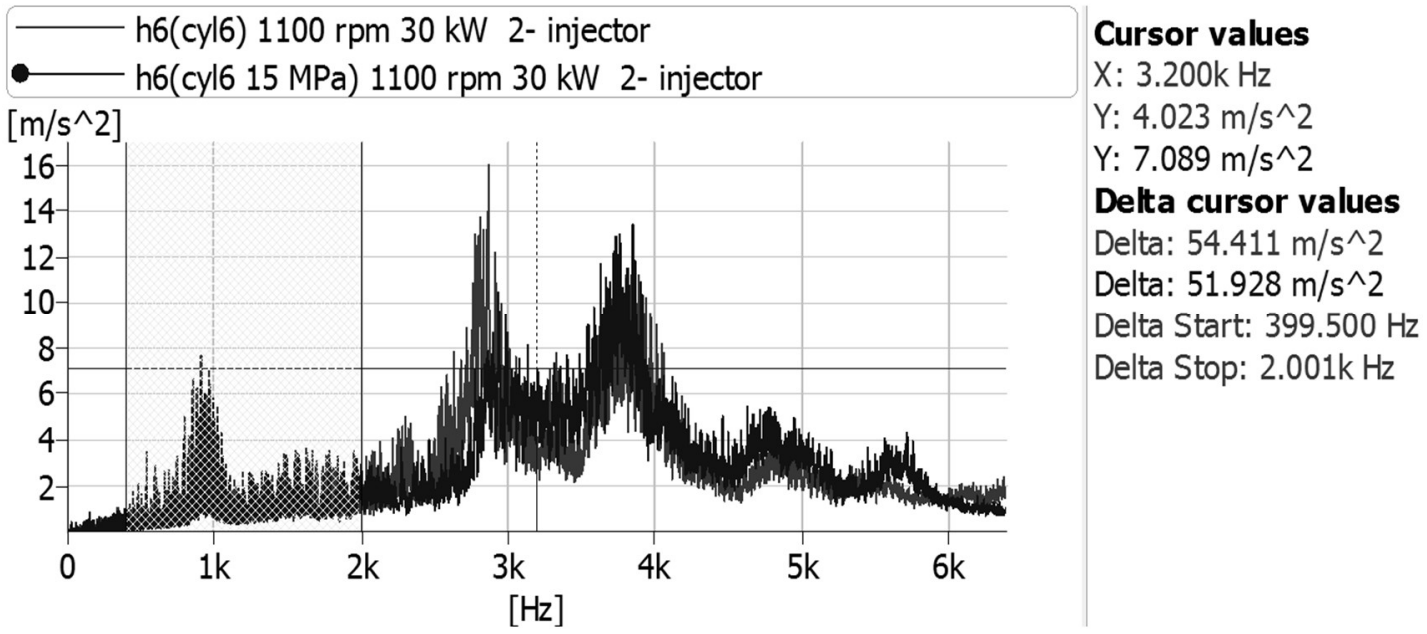

Fig. 10. The vibrations accelerations amplitude-frequency spectra of the injector measured vertically during operating on engine at rotational speed of $1100 \mathrm{rev} / \mathrm{min}$ for two different injection pressures.

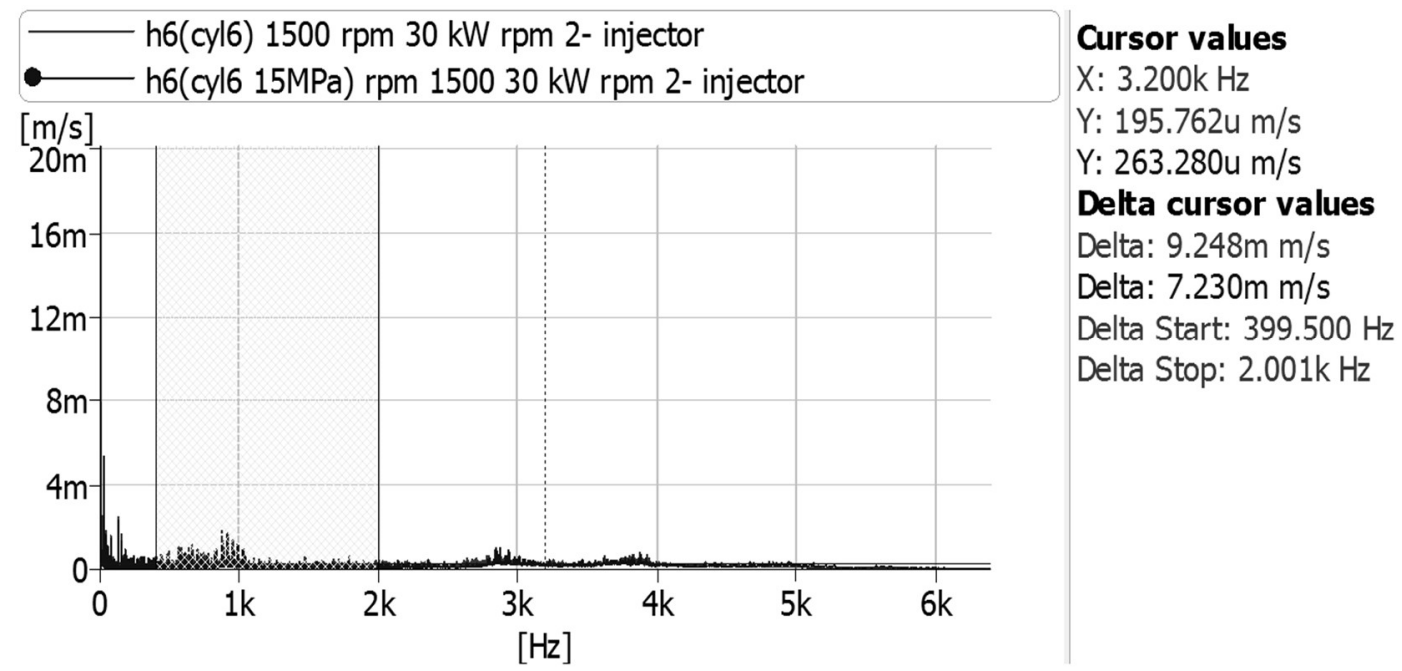

Fig. 11. The vibrations velocities amplitude-frequency spectra of the injector measured vertically during operating on engine at rotational speed of $1500 \mathrm{rev} / \mathrm{min}$ for two different injection pressures.

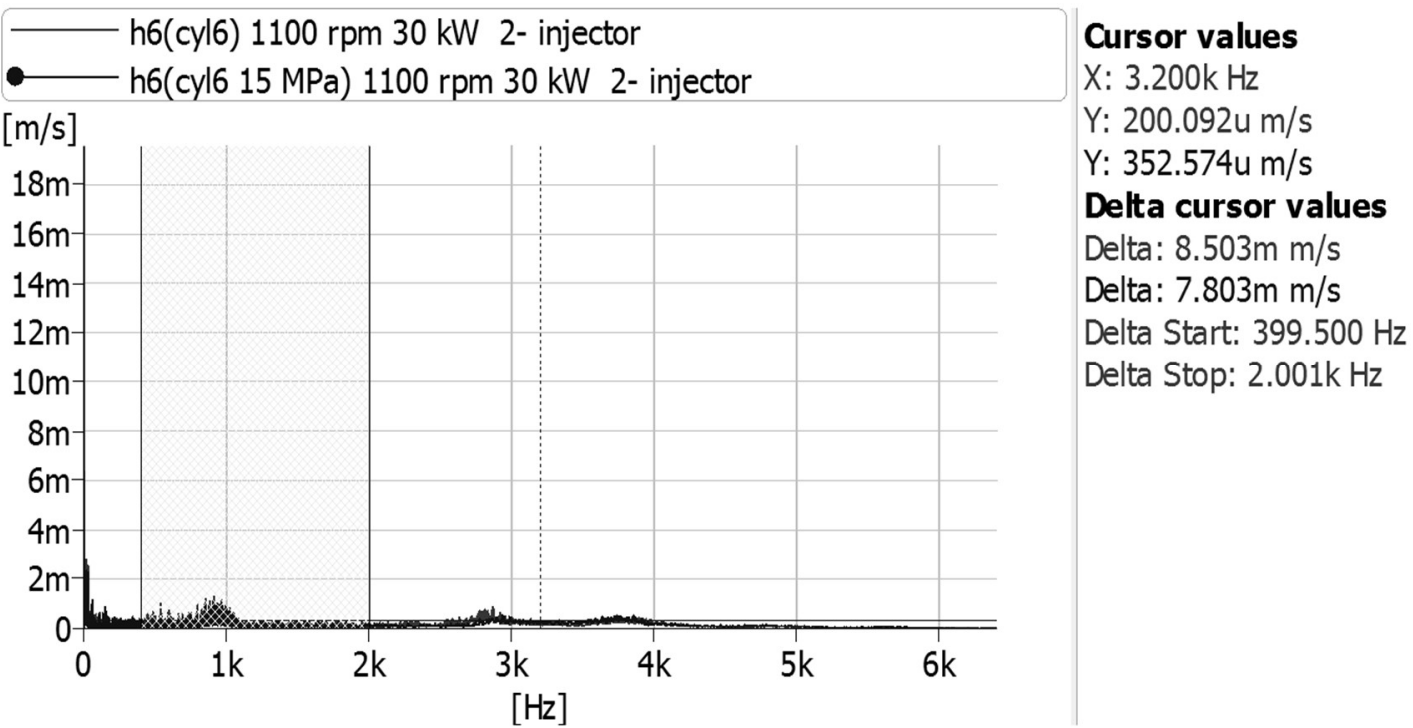

Fig. 12. The vibrations velocities amplitude-frequency spectra of the injector measured vertically during operating on engine at rotational speed of $1100 \mathrm{rev} / \mathrm{min}$ for two different injection pressures. 


\section{CONCLUSION}

The conducted simulations allowed determining the resonant frequency range of the injector. This allows for the determination of the resonant frequency range that is excited during its normal operation. On this basis, passive-active experiment was conducted which revealed the relationship between the injection pressure determined by the injector spring pre-tension and the vibration signals recorded on the injector body. The analysis of the waveforms shows a much more complex nature of the vibration processes occurring during engine operation compared to the vibrations recorded at the injector control station. Lack of access to the results of earlier modal analysis carried out in the MES environment would, therefore, prevent the correct determination of the technical condition of the injectors. The discussed method of diagnosing the technical condition of mechanical fuel injectors still requires a lot of research, but it should be assumed that it will be possible to determine vibration parameters that identify the technical condition of the injector as efficient or inefficient.

\section{REFERENCES}

Antoni, J. Daniere, J. Guillet, F. (2002). Effective vibration analysis of IC engines using cyclostationarity part I: a methodology for condition monitoring. Journal of Sound and Vibration, 257(5), pp. 815-837.

Antoni, J. Daniere, J. Guillet, F. (2002). Effective vibration analysis of IC engines using cyclostationarity part II: new results on the reconstruction of the cylinder pressures. Journal of Sound and Vibration, 257(5), pp. 839-856.

Carlucci, A.P. Chiara, F.F. Laforgia. D. (2006). Analysis of the relation between injection parameter variation and block vibration of an internal combustion diesel engine Journal of Sound and Vibration, 295, pp. 141-164.

Czechyra B. (2008). The injection proces representation in the diesiel engine vibration signal [in polish]: Odwzorowanie procesu wtrysku paliwa w sygnale drganiowym silnika o zapłonie samoczynnym]. Diagnostyka, 4(48), pp. 149-152.

Elamin. F. Gu, F. Ball, A. (2010). Diesel Engine Injector Faults Detection Using Acoustic Emissions Technique. Modern Applied Science, 4(9). pp. 3-13.

Grządziela, A. Kluczyk, M. (2017). High and medium speed marine diesel engines injection installation vibration diagnostics. Diagnostyka, 18(2), pp. 37-42.

Jianmina, L. Yupenga, S. Xiaominga, Y. Shiyongb, X. Lijun, D. (2011). Fuel Injection System Fault Diagnosis Based on Cylinder Head Vibration Signal. Procedia Engineering, 16, pp. 218-223.

Kluczyk, M. Grządziela, A. (2017). Vibration diagnostics of the naval propulsion systems. Scientific Journal of Polish Naval Academy, 1(208),pp.15-29. Lebold, M. Pflumm, S. Banks, J. Bednar, J. Reichard, K. Fischer, K. Stempnik, J. (2012). Detecting injector deactivation failure modes in diesel engines using time and order domain approaches [online] pp. 1-12. https://www.phmsociety.org/sites/ phmsociety.org/files/phm_submission /2012/phmc_12_113.pdf

Monieta, J. (2013). The application in-cylinder pressure course in different domains for diagnostics of medium speed marine diesel engines in operation conditions. Combustion Engines. 154(3), pp. 153-160.

Moshou, D. Natsis, A. Kateris, D. Pantazi, X-E. Kalimanis, I. Gravalos, I. (2014). Fault Detection of Fuel Injectors Based on One-Class Classifiers. Modern Mechanical Engineering. 4, pp. 19-27.

Ranachowski, Z. Bejger, A. (2005). Fault diagnostics of the fuel injection system of a medium power maritime diesel engine with application of acoustic signal. Archives of acoustics. 30(4), pp. 465-472.

Wang, Z. (2009). Study on fault diagnosis of fuel injection based on vibration signal analysis of high-pressure fuel injection pipe. Applied Physics Research, 1(2), pp. 102-106 